\title{
Design of Technical Support System for Retail Company Based on Cloud
}

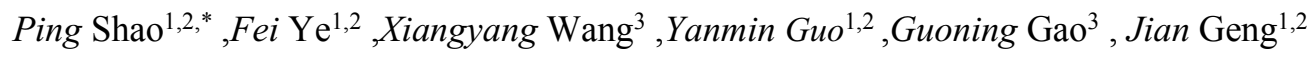 \\ ${ }^{1}$ China Electric Power Research Institute, 210003, China \\ ${ }^{2}$ Electricity Marketing Operation Technology Laboratory of SGCC, Nanjing, 21003, China \\ ${ }^{3}$ Zhejiang Electric Power Trading Center Co., Ltd, Hangzhou, 310037, China
}

\begin{abstract}
With the retail side of the market in China, the sale of electricity companies as a new source of power retail, they participate in the electricity market business. National and local governments subsequently introduced the corresponding policies and rules, the technical support system becomes one of the necessary conditions for the access of the retail company. Retail electricity companies have started the system construction, but has not yet formed a standardized, complete architecture. This paper analyzes the business and data interaction requirements of retail electricity companies, and then designs the functional architecture based on basic application, advanced application and value-added application, and the technical architecture based on "cloud". On this basis, the paper discusses the selection of private cloud, public cloud and mixed cloud model, and the rationalization suggestion of system construction. Which can provide reference for the construction of the technical support system of the domestic retail enterprises.
\end{abstract}

Keywords. power market; cloud; retail electric company; technical support system; system structure

\section{Introduction}

2015, "China Development and Reform Commission on the 9th document" established the current stage of China's power reform direction, the document clearly put forward the "steady promotion of retail electricity side reform, orderly release of retail electricity business to social capital" [1]. In November the same year, the National Development and Reform Commission, Energy Bureau issued a supporting document "on the promotion of retail electricity side of the implementation of the views of reform", in October 2016, issued a document "retail electricity company access and exit management rules", it further clarified the responsibilities and orientation of market members. Requiring the retail company "should have a fixed scale of business with the fixed place of business and power market technical support system and customer service platform" [2]. "Guangdong Province, the implementation of the pilot side of the implementation of the pilot reform program," clearly requires retail companies with user management, trading, billing and other functions of the technical support system [3]. As a result, it becomes one of the most important jobs for retail companies to enter the market that building technical support systems to meet market requirements.

Retail electricity company technical support system is different from the traditional power information system, one is the use of various types of small and medium-sized power users and retail electricity companies internal users; Second, China's electricity market has just opened soon, the business is still in the development stage, Its technical support system is also the side of the construction side to explore, it did not accumulate enough technology; the third is most of the retail electricity companies do not have a strong information technology and operation and maintenance team conditions. Simple to only through the rental cloud system, end users can use including PC, laptop or a smart phone, they can complete the power for the user information exchange and internal operations and other tasks.

"Cloud" appears, is to meet the latest Internet technology development direction, with high reliability, data processing capacity, flexible and scalable and high utilization of equipment advantages [4,5]. Cloud technology has matured in the power industry scheduling, marketing and other specialized systems [6-9], while the retail electrical company technical support system is a completely new information system for the Internet. The majority of small and medium-sized retail electricity companies with cloud technology, they do not bother to support the storage, Internet, servers and other information resources. Energy Internet and large data applications is the future development trend of retail electricity companies [10], only the Internet-facing cloud system architecture have the ability to achieve the future of large data integration, analysis, they are possible to provide users with better energy-packages and energy-solutions. At the same 
time, the cloud architecture is also an information system of operational outsourcing $[11,12]$, it can effectively reduce system construction and operating costs.

This paper analyzes the business requirements of the retail electricity company, and then designs the technical support system architecture of the retail company based on "cloud", and describes in detail the functional architecture, technical architecture and security measures of the system, and finally discusses the "cloud" architecture of the selection, this paper has the characteristics of low cost, high reliability, flexible and easy expansion, and the design idea has been applied in the construction practice of some retail electrician technical support system.

\section{Requirements analysis}

\subsection{Business needs}

Retail electricity market has been more mature in foreign countries, power users, distributed energy owners have been able to participate in the retail electricity market bidding decision, energy efficiency analysis, demand response business $[13,14]$, the retail business is the core needs of the company in China. Electricity customers are the terminal service objects of the retail electricity companies, the electricity as the service carrier, the technical support system as the interactive tool, around the customer's electricity consumption to carry out various services.

China's power purchase transactions mainly in the annual long association and monthly bid trading, retail companies must understand the rules of transaction bidding in order to seize the initiative in the market. It is not only required to manage, track and analyze the purchase contract, but also to support the analysis of the results of the successful bidding of the historical transaction, to automatically generate the next round of market bidding through the auxiliary decision function to achieve the best cost of purchasing power.

The retail business is a power retail business for electricity customers. Customers are the core resources of retail electricity companies, in the fierce competition in the electricity market, the better customer service, increase customer viscosity, which is highly concerned about the content of retail electricity companies. In addition, after further liberalization of the market, retail companies must also face a series of problems caused by the explosive growth of power customers, how to effectively maintain customer information, rapid response to customer needs and improve efficiency, will become a retail company must consider problem.

The new round of power reform not only revitalize the retail electricity market, but also to promote integration with other related industries, retail companies to provide diversified value-added services to open the door. Energy efficiency control, demand side management, electrician on behalf of Victoria and so on are value-added services, these value-added services will be launched around the sale of electricity business, reflecting the value of retail professional services [10].

Therefore, the technical support system should be through information technology means to analyze the customer's electrical characteristics, rich design of electricity packages, to provide users with energy-saving recommendations such as personalized care service. These seemingly trivial features can greatly enhance the power of customers electricity experience and retail electricity service quality.

\subsection{Data interaction requirements}

To meet business needs, retail companies need to interact with third-party systems. Such as from the power trading center to obtain open transaction data, to the power trading center to provide quotations, retail contract reporting, etc. To obtain the power acquisition data from the distribution system; to obtain the grid model data from the dispatch system for bid decision, etc., these data will effectively support the normal operation of the retail electricity company business.

The current situation, regardless of the State Grid or the Southern Power Grid within the scope of the power exchange center at all levels failed to provide for these data needs of the interactive interface. It is reported that the relevant units have been in the lead in the development of trading platform and retail electricity company technical support system information industry standards. The mechanism will not only meet the basic needs of transaction settlement in data quality and timing, but also will help improve the overall quality of service in the retail electricity market [15]. Therefore, when the conditions are met in the external policy and automation, the system should provide the ability to dock data with the trading platform to enhance the efficiency of power purchase and sale of electricity business.

\section{System function architecture}

The technical support system mainly supports the retail electric companies to carry out bid trading and electricity sales service, at the same time for its terminal power customers to provide service window, to achieve the twobusiness interaction. System function architecture shown in Figure 1, according to the user's classification of business needs, can be divided into basic applications, advanced applications and value-added services in two parts, a total of 10 functional modules.

\subsection{Basic application}

\subsubsection{Customer file management}

The customer model is the basic information for the sale of electricity. China's power customers to enter the market is gradually released in accordance with the voltage level, so the minimum size of the business should be "electric 
card number + voltage level" of the particle size. In addition, the retail electricity companies are also involved in the wholesale market power purchase business, the establishment of trading institutions, power generation companies, transmission and distribution companies such as the market model for signing contracts, settlement and so on. This part of the model should inherit the transaction platform IEC 62325 model standard [16].

\subsubsection{Customer service}

There is a lot of information interaction and business interaction between retail electricity companies and power customers. Should use the Internet mature technology, through the online business hall, mobile APP, WeChat and other means for customers to facilitate and efficient means of interaction.

Customer service includes public and private information. Public information includes corporate announcements, market information releases, policies and regulations, price-packages, etc., which are open to all visitors. Private information includes historical transactions, electricity, electricity, contract signing, electricity demand, information subscription, etc., these are the customer's own private information.

Web page should pay attention to the comprehensiveness of information, the convenience of operation, through visual analysis, report display, etc., it provides users with intuitive information display, and support users to download and export, mobile APP and WeChat public number is to provide users with similar Simplified version of the function, user-friendly time to grasp the market information and their own electricity data.

\subsubsection{Retail electricity management}

It is responsible for the management and power customers related to the contract, electricity data, electricity billing and so on. The electricity contract is the legal agreement between the retail electricity company and the electricity customer, which is the basis of the electricity settlement. Specific features include: contract editor, annual contract automatically assigned to the month, the contract text management, electronic contract generation, contract changes and so on. Contract implementation process, combined with the actual customer electricity situation, the system provides the implementation of the contract tracking, deviation from the warning, risk analysis and other information, to give customers a comprehensive information to remind. The system provides monthly settlement function, according to the energy meter count or electricity customers to fill the actual electricity consumption, combined with the deviation assessment requirements, and then electricity calculation, and submitted to the power trading platform.

\subsubsection{Power management}

Another important function of the retail electricity company is to carry out electricity purchase activities in the wholesale market. Features include purchase contract management, power purchase plan, power purchase settlement and earnings analysis.

Purchase contract management: including power generation companies purchase contracts, transfer contracts, transmission and distribution service contracts, including bilateral and centralized, and so on. Specific features include contract editing, contract decomposition to month, contract generation documents, electronic signatures, etc., it is similar to the sale of electricity contract, but the nature of the contract is concerned about the difference.

Transaction plan management: for the new round of annual agreements, monthly concentration of transactions, as well as the day-ahead and real-time market transactions, through the collection of electricity demand data and forecast electricity data, and finally the formation of transaction plan management.

Power purchase settlement and benefit analysis: After the implementation of the monthly, according to the actual electricity data, combined with the deviation assessment requirements, the purchase side of the contract to settle, and with the electricity trading center settlement data to check. At the same time carry out profit analysis, statistical balance of sales, used to guide the next power transaction quotes.

\subsubsection{Price-Packages}

Electricity price is the most important means for retail customers to attract customers. The system should support the management of various types of price-packages, including fixed price model, divided into models, fixed price plus points and other mixed mode. With the rules of the market, the supply and demand situation often changes, so the functional design needs to consider its scalability.

In addition to electricity prices, the system should also provide the transmission and distribution price, capacity price, collection price and other catalog price maintenance, in order to help customers, complete the accounting of various types of electricity.

\subsubsection{Distribution management}

For the distribution and retail electricity in one company, according to No. 5 views, once the power distribution business, you need to bear the measurement, charges and other corresponding functions. Therefore, for such retail companies, the system should provide electricity collection, electricity billing, charge management, equipment operation and maintenance, asset management, and other original power companies provided by the support services. Due to the high real-time requirements of distribution network monitoring applications, it is recommended to adopt the independent system to realize the data integration with the system. Once the equipment failure affects the electricity, the information can inform the customers in time. In addition, the system can also provide electrical services, new equipment, industry expansion and other functions, the system provides value- 
added services for power customers, agents and all customer-related business and activities.

\subsection{Advanced applications and value - added service applications}

\subsubsection{Auxiliary decision}

The market will run for some time will gradually mature, the rules are relatively stable, but also accumulated some historical data, which support the auxiliary decisionmaking applications. Features include: load forecasting, bid-assisted decision making, power-assisted decision making, and so on.

Load Forecasting. Based on the customer's history of electricity, forecast the future period of electricity data. The system can be predicted for a single customer or full caliber. Time covers the annual and monthly, the future can be extended to a few days ago or even to the hour. Load factors cover data closely related to customers, such as production planning, order status, maintenance plans, industry factors, holiday settings and so on.

Bid support decision. In the submission of a new round of power demand before the need to combine the power purchase contract has been signed, the future of electricity forecast, the system automatically calculates the electricity gap. At the same time, according to the electricity sales contract price, the company operating costs and estimated profits, the system automatically calculate the upper limit of the purchase price. The measured data is used as a reference limit for the price declaration. In addition, the system through the analysis and prediction of other market members of the offer behavior, to carry out business simulation, or directly predict the market marginal price and so on.

Retail Electricity Assist Decision. In the power user side, through the customer history data mining analysis, the formation of the customer's typical electricity curve, and have similar business habits of customers clustering, the formation of customer classification. By analyzing the different customer categories and price-packages, the system to help retail companies to develop a better pricepackages.

\subsubsection{Micro-services}

In the future, retail companies can provide the price reduction trend gradually convergence, its competitiveness will be more and more reflected in the value-added services. Features include micro-network equipment management, measurement and settlement, energy storage equipment management. The operation of the microgrid can be achieved by a separate system, but requires data integration with the system.

\subsubsection{Demand response}

With the advancement of the spot market, demand response through real-time electricity prices to guide the market members of the electricity behavior. Features include resource management, project management, customer participation willingness collection, DR instruction issuance, real-time load monitoring, revenue calculation, demand response assessment. Retail electricity companies to guide customers through the short-term electricity behavior, to reduce the power load for a certain period of time, it can guarantee the stable operation of the grid and inhibit the rise in electricity prices, and ultimately to achieve revenue sharing with the user.

\subsubsection{Energy efficiency services}

Retail companies can enhance customer stickiness through energy efficiency services. The system can provide electricity monitoring, energy efficiency analysis and benchmarking, energy efficiency potential analysis, analysis, etc., the system can help retail companies understand the entire park and the various equipment within the enterprise energy situation, and put forward energy-saving recommendations.

\section{Technology Architecture}

The technical architecture is the underlying architecture in the TPS technical support system, which describes how to build an IT runtime environment to support data and functional architectures to ensure that the business is working properly. This section will focus on the technical requirements of the retail company to support the software system and propose an adaptive technology architecture.

In order to solve these problems and challenges, the use of virtualization technology, load balancing, hot backup redundancy and network storage and distributed technology [17-19], designed based on "cloud" technology architecture technical support system. As shown in Figure 2, the technical architecture is divided into four layers: IaaS (Infrastructure as a Service), PaaS (Platform as a Service) SaaS (Software as a Service) And presentation layers.

Infrastructure layer IaaS, implemented by a third-party cloud computing platform or a retail company's existing cloud resources for virtualization. Through the virtualization platform, the host, storage devices and network equipment and other hardware resources for unified management. The PaaS platform layer is a collection of platform resources that are versatile and reusable. The platform layer provides software development kits (SDKs) and application programming interfaces (APIs) for the upper-tier power-hungry applications to develop a test environment that forms the operating environment for the sales application business. SaaS software application layer is a collection of application software on "cloud". All applications run as micro service in the service platform, according to the degree of hot and cold interface of the gateway. The first three layers are essentially the integration of resources, respectively, IT infrastructure, development and operation platform, application software as a service, the user ondemand use [20]. The top layer of the presentation layer to 
provide Web, App, WeChat and multi-carrier display terminal, etc., they are through the Restful-API and backend interaction, to achieve front and back separation.

"Cloud" architecture reduce the difficulty of enterprise deployment, saving hardware costs, improve system scalability and other advantages, but in the deployment, there are some concerns in the power customer electricity information and enterprise core data leakage and system operation security. the system needs to use such as system isolation and authority control, identity authentication, communication and data encryption [21] and other technical measures to protect the security of the system. Specific measures are as follows:

(1) system isolation and permission control. The SaaS layer of the electricity application and the system operating space of the PaaS layer of the platform are isolated, and the IaaS layer virtual resource monitoring software is used for real-time monitoring analysis. [22] The customer account of the retail electricity company can only access its internal application data table, the power user's access account can only access the application data for the power user, the two applications through the interactive service synchronization data. Through the logical isolation of two relatively independent WEB services, and ultimately to ensure the security of the system through the logical security boundaries.

(2) identity authentication. According to the different use of the system, we will be divided into three categories: the first category is the retail electricity company agent power users, the main access to the power of the user's online business hall and APP applications; the second category is the retail company's ordinary users, The main access to the system purchase and sale of electricity-related basic application functions; the third category is the retail electricity company's core users, the main use of bidding decision-making and operating KPI indicators analysis applications. The first two types of users using the account password + mobile phone dynamic password mainstream authentication, user-friendly quick access to the system. The third type of users because of the confidentiality of business functions related to access, then the use of triple joint authentication, including CA digital certificate + account password + SMS password.

(3) communication security. Retail companies and their agents all need to access the Internet through the Internet technical support system, in order to protect the stability and security of the network, the system uses at least two of the Internet access channel. At the same time, the website using Hyper Text Transfer Protocol over Secure Socket Layer protocol to prevent data in the transmission process will not be stolen, change, to ensure data integrity. In addition, the system can resist DDos (Distributed Denial of Service) services through the purchase of network hardware devices or third-party services provided, it can ensure that the system communication and service is stable and reliable.

(4) data encryption. The system uses the PaaS layer to deploy Transparent Data Encryption [23]. The system performs real-time $\mathrm{I} / \mathrm{O}$ encryption and decryption on application data. It ensures that the entire database is protected by writing encryption, reading and decrypting, protecting data files and logs file. In addition, the system can also encrypt the sensitive data of the retail company through the hardware encryption machine or software encryption algorithm, such as the system of auction transactions, contract price, settlement of electricity and other confidential data using a variety of encryption algorithms to encrypt operations [24].

\section{Discussion on Architecture Selection and System Construction}

In a variety of "cloud" architectures, such as private clouds, public clouds and hybrid clouds, they have advantages and disadvantages in terms of data privacy, flexibility, load capacity, technical difficulty, and operational costs. As shown in Table 1, private cloud applications and operational data in the self-built system, the privacy of its data has a higher protection, but because it can only be deployed on its own server, not only the existence of higher investment costs, And the system expansion is also limited; the public cloud has a better scale effect by constantly leasing new equipment to deal with uncertain load requirements, the underlying system and network operation and maintenance can be provided by the public cloud manufacturers, investment and operating costs lower; Mix-Cloud is a private cloud + public cloud mixed architecture that has higher data privacy and flexibility than public clouds. $[25,26]$ The system offers highly scalable, low-cost, high-security services. When there is a high workload demand, the company can share the high load by renting the public cloud.

Table 1. Features comparison between three Clouds Structur

\begin{tabular}{|c|c|c|c|}
\hline Characteristic & $\begin{array}{c}\text { Private } \\
\text { cloud }\end{array}$ & $\begin{array}{c}\text { Public } \\
\text { cloud }\end{array}$ & $\begin{array}{c}\text { Mixed } \\
\text { cloud }\end{array}$ \\
\hline Data privacy & Higher & Medium & High \\
\hline Flexibility & Medium & Higher & Higher \\
\hline Reliability & High & High & Higher \\
\hline Load Capacity & Medium & Higher & High \\
\hline $\begin{array}{c}\text { Technical } \\
\text { Realization }\end{array}$ & Medium & Low & High \\
\hline $\begin{array}{c}\text { Cost of } \\
\text { Investment }\end{array}$ & Higher & Low & High \\
\hline $\begin{array}{c}\text { Operating } \\
\text { Costs }\end{array}$ & Higher & Low & High \\
\hline
\end{tabular}

Therefore, the selection of the "cloud" architecture of the retail technical support system needs to be considered from its own scale, system flexibility, technical difficulty and operating cost. Large-scale retail electricity companies generally have a strong economic and technical strength, the proposed hybrid cloud architecture system; mediumscale retail electricity companies and economic strength is good, high privacy requirements of the data, the proposed use of private cloud architecture; other Small-scale retail electricity companies recommend using public cloud to build their own technical support system.

\section{Conclusion}


This paper analyzes the business requirements of the retail technical support system and designs the technical support system architecture of the retail company based on "cloud", which can be flexibly selected according to the demand. The architecture of the technology to achieve operational flexibility, to meet the current market requirements and future development needs. At the same time, this paper analyzes the characteristics of cloud, public cloud and mixed cloud, and so on, according to the different requirements of various types of retail electric companies in terms of economy, technology and their own scale, and discuss the rationalization proposal of the system step by step construction. The technical support system architecture of the retail electricity company based on the cloud is a positive attempt to cater to the Internet + , the research results can provide reference for the design of the technical support system of the retail companies.

Acknowledgment: This work was supported by the Science and Technology Project of State Grid Corporation of China under Grant DZ71-17-019.

\section{References}

1. The CPC Central Committee and State Council. Zhong Fa [2015] No. 9 on further deepening the power system reform a number of opinions [Z] .2015-3-15

2. China National Development and Reform Commission National Energy Board. Development and Reform [2016] No. 2120 No. 0 Recycling and withdrawal management of retail companies [Z] .2016-10-11

3. Guangdong Provincial Development and Reform Commission Guangdong Provincial Economic and Information Commission National Energy Bureau of the South Authority. Guangdong Development and Reform Energy [2017] No. 48 Guangdong Province, the implementation of electricity sales reform pilot program [Z] .2017-1-19

4. WANG Dewen, SONG Yaqi, ZHU Yongli.Information Platform of Smart Grid Based on Cloud Computing[J].Automation of Electric Power Systems,2010,34(22):7-12.

5. WANG Dewen,LIU Yang.A Task Scheduling Strategy of Cloud Data Center in Electric Power Corporation $[\mathrm{J}]$.Automation of Electric Power Systems,2014,38(8):61-66.

6. YAO Jianguo,YANG Shengchun,SHAN Maohua.Reflections on Operation Supporting System Architecture for Future Interconnected Power Grid[J].Automation of Electric Power Systems,2013,37(21):52-59.

7. ZHAO Junhua1, WEN Fushuan1, XUE Yusheng2 , LIN Zhenzhil.Cloud Computing: Implementing an Essential Computing Platform for
Future Power Systems[J].Automation of Electric Power Systems,2010,34(15):1-8.

8. QU Zhaoyang,CHEN Shuai,YANG Fan, et al.An Attribute Reducing Method for Electric Power Big Data Preprocessing Based on Cloud Computing Technology[J].Automation of Electric Power Systems,2014,38(8):67-71.

9. Li Dan, Shen Hao, Sun Wei. Exploration and practice of "Internet plus electric power marketing"mode [J]. Power demand side management, 2016, 18 (z1).

10. Zang Ningning. Power System Reform Under The Sale Of Electricity Companies How To Build A Marketing System [J]. China Energy, 2016, 38 (4): 44-47.

11. Zheng Guangcheng. An Soa Cloud Service Platform Architecture Research And Application [J]. Computer Applications and Software, 2011, 28(12):228-231.

12. Liang Shuang. Design and realization of cloud computing framework model based on SOA [J]. Computer Engineering and Applications, 2011, 47 (35): 92-94

13. MA Li,ZHANG,Xiaoxuan,WEI Zhe. Experience and Enlightenment of Power Sales Side Market Liberalization in France [J]. Southern Power System Technology, 2015, 9(8):9-12.

14. ZHANG Xiaoxuan,XUE Song,YANG $\mathrm{Su}$, et al.International Experience and Lessons in Power Sales Side Market Liberalization[J].Automation of Electric Power Systems,2016,40(9):1-8.

15. BAI Yang,XIE Le,XIA Qing, et al.Institutional Design of Chinese Retail Electricity Market Reform and Related Suggestions[J].Automation of Electric Power Systems,2015,39(14):1-7.

16. ZHENG Yaxian,YANG Zhenglin,XUE Bike, et al.Latest Development of International Electricity Market Standards IEC 62325[J].Automation of Electric Power Systems,2015,39(15):1-8.

17. He Hengjing, Zhao Wei, Huang Songling, Wang Qing. Research on the application of cloud computing in power user electric energy data acquisition system [J]. Electrical Measurement \& Instrumentation, 2016, 53(1): 1-7.

18. Armbrust M, Fox A, Griffith R, et al. A view of cloud computing[J]. Communications of the ACM, 2010, 53(4): 50-58.

19. Rittinghouse J W, Ransome J F. Cloud computing: implementation, management, and security[M]. CRC press, 2016.

20. WANG Dewen, SONG Yaqi, ZHU Yongli.Information Platform of Smart Grid Based on Cloud Computing[J].Automation of Electric Power Systems,2010,34(22):7-12.

21. FENG Chao-Sheng, QIN Zhi-Guang[2], YUAN Ding[3]. Techniques of Secure Storage for Cloud Data [J]. Chinese Journal of Computers, 2015, 38(1): 150-163. 
22. LONG Feng, WEN Zhonghua, WANG Quan, WANG Jinzong. User privacy protection against providers in public cloud computing [J]. Computer Engineering and Applications, 2015, 51(23):106-111.

23. Deshmukh D, Pasha A, Qureshi D. Transparent Data Encryption--Solution for Security of Database Contents[J]. arXiv preprint arXiv:1303.0418, 2013.

24. Ali cloud. Ali cloud data security white paper [EB / OL].
Https://help.aliyun.com/knowledge_detail/42566.ht $\mathrm{ml}$.

25. Zhang Zi-lang. The Research on Architecture of Enterprise Information Platform Based on Hybrid Cloud $[\mathrm{J}]$. Information Security and Technology,2014,(04):60-65.

26. Huang Dongmei, Du Yanling, He Qi. Migration Algorithm for Big Marine Data in Hybrid Cloud Storage [J]. Journal of Computer Research and Development,2014,(01):199-205.

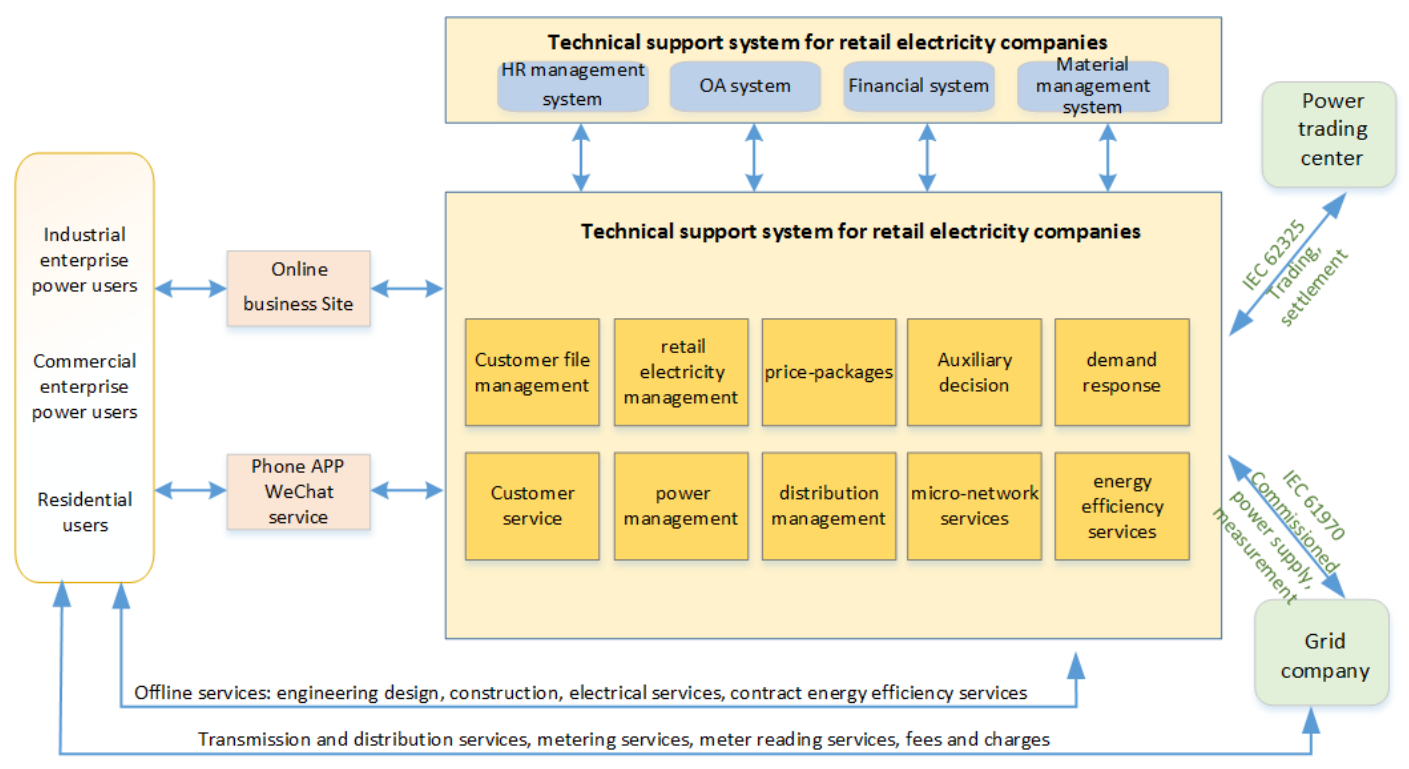

Fig. 1. System functions architecture of retail electricity company

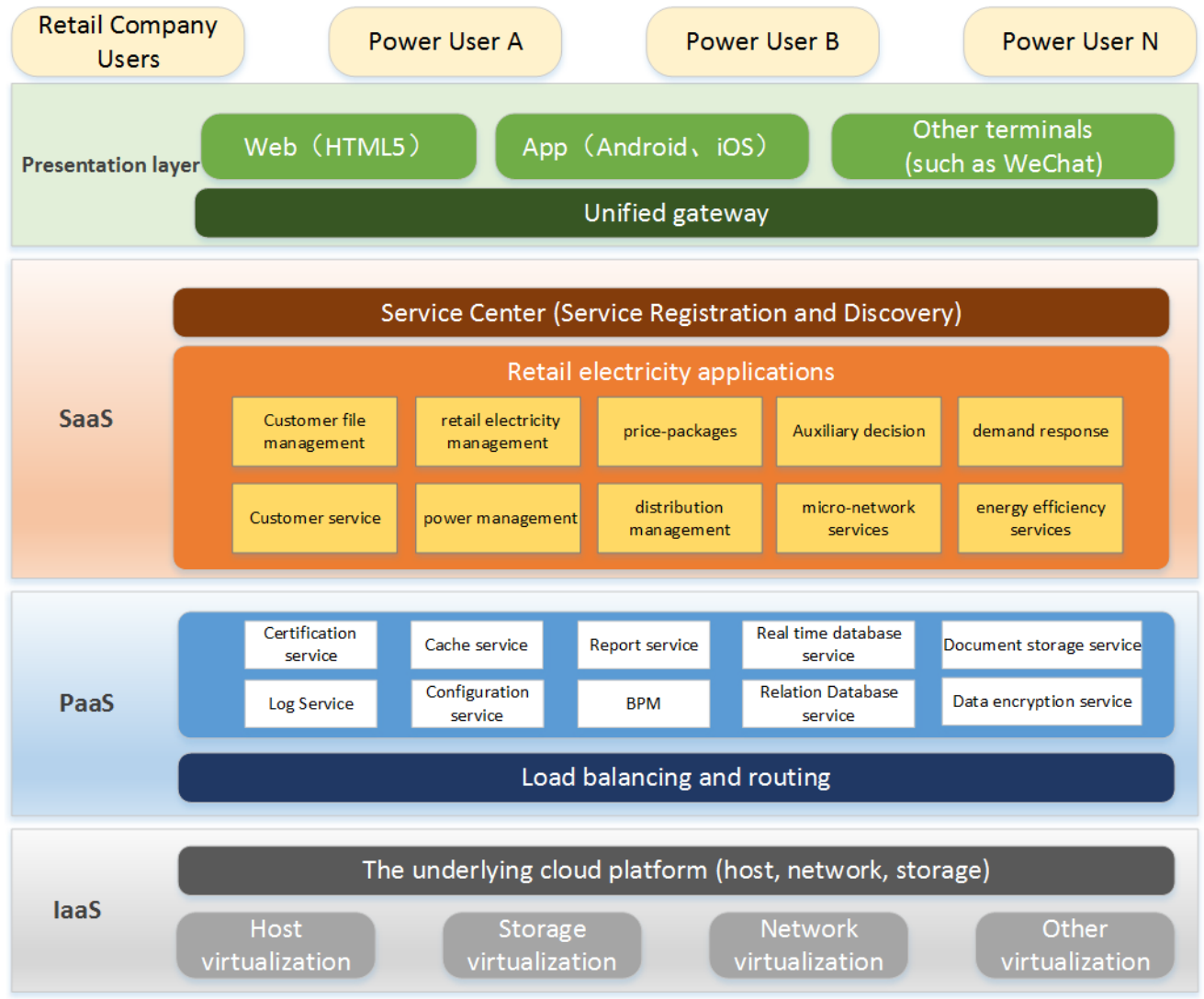

Fig. 2. System technical architecture of retail electricity company 